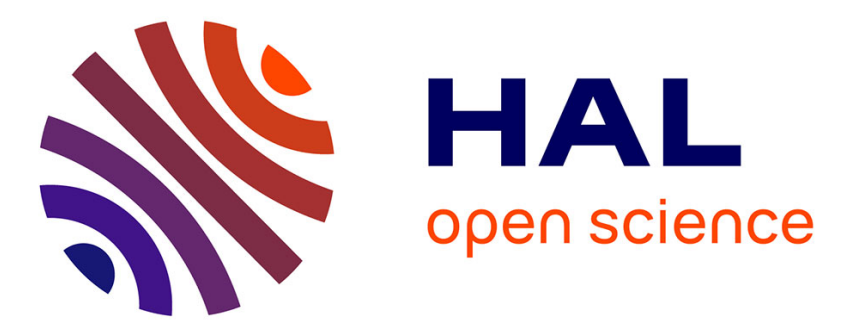

\title{
Non-linearized amplifier and advanced mitigation techniques: DVB-S2X spectral efficiency improvement
}

Jean-Alain Lucciardi, Philippe Potier, Guillaume Buscarlet, Fatima Barrami, Gilles Mesnager

\section{- To cite this version:}

Jean-Alain Lucciardi, Philippe Potier, Guillaume Buscarlet, Fatima Barrami, Gilles Mesnager. Nonlinearized amplifier and advanced mitigation techniques: DVB-S2X spectral efficiency improvement. IEEE GLOBECOM 2017, Dec 2017, Singapore, Singapore. pp.1-7. hal-02651509

\section{HAL Id: hal-02651509 \\ https://hal.science/hal-02651509}

Submitted on 29 May 2020

HAL is a multi-disciplinary open access archive for the deposit and dissemination of scientific research documents, whether they are published or not. The documents may come from teaching and research institutions in France or abroad, or from public or private research centers.
L'archive ouverte pluridisciplinaire HAL, est destinée au dépôt et à la diffusion de documents scientifiques de niveau recherche, publiés ou non, émanant des établissements d'enseignement et de recherche français ou étrangers, des laboratoires publics ou privés. 


\section{OATAO}

Open Archive Toulouse Archive Ouverte

Open Archive Toulouse Archive Ouverte

OATAO is an open access repository that collects the work of Toulouse researchers and makes it freely available over the web where possible

This is an author's version published in: http://oatao.univ-toulouse.fr/22318

\section{Official URL:}

https://doi.org/10.1109/GLOCOM.2017.8255091

\section{To cite this version:}

Lucciardi, Jean-Alain and Potier, Philippe and Buscarlet, Guillaume and Barrami, Fatima and Mesnager, Gilles Non-linearized amplifier and advanced mitigation techniques: DVB-S2X spectral efficiency improvement. (2018) In: IEEE GLOBECOM 2017, 4 December 2017 - 8 December 2017 (Singapore).

Any correspondence concerning this service should be sent to the repository administrator: tech-oatao@listes-diff.inp-toulouse.fr 


\title{
Non-Linearized Amplifier and Advanced Mitigation Techniques: DVB-S2X Spectral Efficiency Improvement
}

\author{
Jean-Alain Lucciardi*, Philippe Potier *, Guillaume Buscarlet*, Fatima Barrami* and Gilles Mesnager* \\ * Embedded Systems, IRT Saint-Exupery, Toulouse, France \\ Email: forename.name@irt-saintexupery.com
}

\begin{abstract}
The latest standardization DVB-S2X increases the achievable spectral efficiency of the satellite communications by around $15 \%$ in AWGN channel. In order to benefit from those improvements, the strong non-linear distortions introduced by the payload have to be overcome, mostly taking high back-off on the amplifier operation point. Nowadays, on-board amplifiers are linearized before being deployed, allowing low-complexity transmitters and receivers at the detriment of the payload's cost and reduced energy efficiency. In this paper, various techniques are investigated for the purpose of spectral efficiency improvement while releasing the amplifier linearization constraint. Iterative pre-distortion at the transmitter, turbo-equalization at the receiver and appropriate waveforms for transmission through non-linearized payload appear as strong candidates considering the results of this study.
\end{abstract}

\section{INTRODUCTION}

The increased achievable spectral efficiency in satellite communications offered by the new standard DVB-S2X [1] is mostly due to two innovations. On one hand, tighter rolloff factors, 0.1 and 0.05 , increase the capacity offered in the bandwidth, decreasing the rejection zone of the shaping filter. On the other hand, higher Amplitude and Phase Shift Keying (APSK) modulation orders (64-APSK, 128-APSK and 256APSK) are incorporated. The announced gains are computed for a linear AWGN channel, without taking into account the payload's memory effect whereas the two aforementioned innovations decrease the robustness to non-linearity. In order to benefit from the potential of the satellite standard evolution, linearized amplifiers are paired with straightforward Least-Mean-Square (LMS) equalizers [2]. Subsequently, the achievable transmission performance does not achieve the full potential of the standard.

A previous paper from Piemontese et al. [3], published before the DVB-S2X update, investigated different advanced pre-distorters and receivers for spectral efficiency increase in non-linear satellite channel. Significant gains were noticed thanks to pre-distortion, Time-Frequency Packing (TFP), Volterra based equalization and bandwidth optimization. Although demonstrating the interest of advanced receivers for transmission rate increase, the comparison did not take into account the aforementioned DVB-S2X innovations and the linearized amplifier option of current satellite channel. The objective of this paper is to demonstrate that with appropriate mitigation techniques, we can get closer to the DVB-S2X full potential without amplifier linearization.

In [4], an efficient pre-distortion technique, based on the socalled Small Variation Algorithm (SVA), has been proposed to mitigate the effects of the distortions induced by the satellite payload High Power Amplifier (HPA) and channelizing filters (IMUX, OMUX) in DVB-S2 transmissions. Improvement of the system spectral efficiency has been assessed for the higher DVB-S2 modulation scheme (i.e. 32APSK), and compared to State-Of-the-Art (SOA) pre-distorters. This study clearly demonstrates that SVA outperforms SOA pre-distorters, but at the price of an increased complexity. Preliminary approximations have been proposed to reduce that complexity in DVBS2 context. In this paper, SVA optimization is reconsidered to allow its implementation in future DVB-S2X wide-band modulators. The achievable gain of the system spectral efficiency is assessed over a wide SNR range, optimizing the transmitting symbol rate and the HPA operating back-off.

Advanced receivers are also investigated in this paper. In [5], the Volterra model for non-linear channel modeling through Volterra kernels is depicted. Non-linear [6] and linear [7] Volterra based detectors can be implemented at the receiver for spectral efficiency improvement in strong non-linear channel context. Moreover, such advanced receivers allow an increase of the transmission rate through faster-than-Nyquist (FTN) signaling [3]. Its spectral efficiency improvement is obtained with no complexity increase compared to Nyquist signaling when strong non-linear distortion is considered with Volterra model.

The Volterra based techniques investigated in this paper have the non-negligible drawback of complexity increase. In this sense, waveforms implementing frequency domain equalization which allow low-complexity receivers, Single CarrierOrthogonal Frequency Division Multiplexing (SC-OFDM) signaling and Extended Weighted (EW-SC-OFDM) signaling are investigated.

Finally, each advanced technique is investigated with another degree of freedom : the rate optimization. The allocated bandwidth is fixed but the transmission rate can be increased, allowing use in the selective zone of the different filters.

The paper is organized as follows. Section II briefly reviews the comparison framework's channel, from the transmitter to the receiver going through the payload. Section III introduces 
the different aforementioned techniques offering spectral efficiency increase in non-linear context. Finally, in section IV, the benefits of the different methods are deeply investigated, depending on the operating SNR.

\section{CONTEXT OF COMPARISON}

In this section, the evaluation framework is detailed, ensuring a fair comparison of the different methods performance with regard to a typical DVB-S2X transmission.

\section{A. Rate optimization regarding the transmission bandwidth}

The transponder allocated bandwidth is $B=40 \mathrm{MHz}$. The shaping filter $h_{e}$ is a Square Root Raised Cosine (SRRC) with a roll-off factor $\beta=0.10$ and a conventional rate $R_{s}=36$ MBauds. At the receiver, the matched filter $h_{r}(t)=h_{e}^{*}(-t)$ is applied. This template involves the possibility of transmission with higher rates in the fixed bandwidth, increasing the asymptotic spectral efficiency but not fulfilling the criterion $B=R_{s}(1+\beta)$ for shaping filter. Then, at the receiver, the whole signal is not recovered, leading to a capacity loss and a higher channel selectivity. Depending on the technique and the SNR, the rate expansion can increase or decrease the capacity, leading to an optimization for each technique.

\section{B. Satellite channel - Payload description}

The considered payload is transparent and the transmission scheme is a Single Carrier Per Channel (SCPC) one. Onboard the satellite, an Input Multiplexer (IMUX) bandpass filter selects the sub-band to amplify. Then, the HPA introduces non-linear distortion in the channel model. Finally, an Output Multiplexer (OMUX) bandpass filter removes the out-of-band interferences produced by the power amplifier.

The AM/AM characteristics of the two considered types of HPA are given in Figure 1-a.

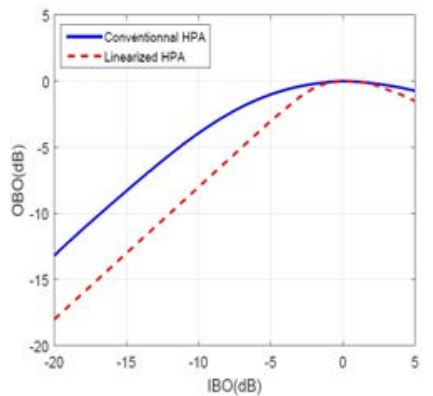

(a)

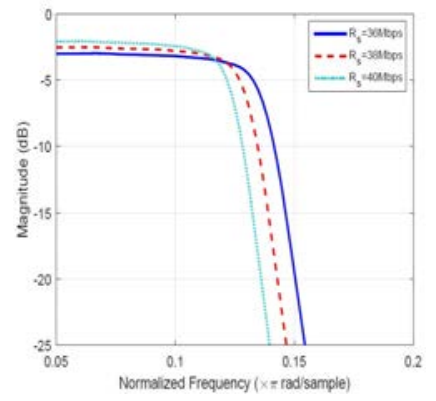

(b)
Fig. 1: DVB-S2X channel components: (a) the HPA OBO as function of the IBO) (b) OMUX frequency response

A major difference between the two amplifiers is highlighted: the conventional amplifier has a higher energy efficiency than the linearized amplifier. In Figure 1-b, the OMUX frequency response is given considering the possible rate enhancement detailed in II-A. The usual transmission scheme in DVB-S2X context is illustrated in Figure 2.

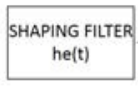

TRANSMITTER

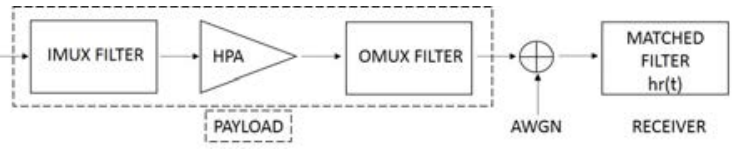

Fig. 2: Usual transmission scheme

\section{EXIT (EXtrinsic Information Transfer) charts}

In order to evaluate the asymptotic performance for the different transmission schemes, the achievable spectral efficiency is evaluated based on EXIT charts computations [8]. To this end, the achievable coding rate is first evaluated using the area theorem (area under the obtained EXIT curve). Even if the area theorem is only proved for the binary erasure channel, it provides good approximation for other types of channels. The area under EXIT curves gives the highest achievable coding rate suitable for the iterative detection. Then, the achievable spectral efficiency is deduced as a function of the SNR [9].

\section{AdVANCED Mitigation TECHNIQUES}

In order to overcome the distortion introduced by the conventional amplifier depicted in Figure 1, many advanced transmission techniques are considered. First of all, the relevance of different pre-distortion methods implemented at the transmitter is discussed in sub-section III-A. Subsequently, advanced Volterra based detectors are investigated, challenging Minimum-Mean-Square-Error (MMSE) and Maximum-APosteriori (MAP) equalizers in sub-section III-B. The tradeoff between complexity and performance increase is debated. Then, FTN signaling paired with advanced Volterra based MAP detector is investigated in III-C for spectral efficiency enhancement. Last but not least, SC-OFDM waveform coupled with low-complexity equalization in frequency domain is detailed in III-D.

\section{A. Pre-distortion methods}

In [4], the authors have proposed a new iterative predistortion algorithm, called SVA. Their study has shown that the performance improvement brought by SVA algorithm represents several $\mathrm{dB}$ on the $\mathrm{MSE}$ and up to $1.5 \mathrm{~dB}$ on the link budget with 32-APSK modulation, compared to state-ofthe-art pre-distorters based on memory polynomials [10] and look-up tables [11]. Basically, SVA consists in pre-distorting a block of $L$ transmitted symbols to minimize the Euclidian distance between the transmitted symbols $s$ and the received symbols $\boldsymbol{y}$

$$
\epsilon_{k, j}=\boldsymbol{y}-\boldsymbol{s}
$$

The pre-distorted symbols $x_{k, j}$ are updated at each step $j(j=1, \ldots, L$ assuming a block of $L$ symbols $)$ of each $k$ iteration based on a linear approximation of the channel output variation $F_{k, j}^{L_{i n}}$ :

$$
x_{k, j}=x_{k, j-1}+\Delta_{k, j}^{L_{i n}}
$$

where $\Delta_{k, j}^{L_{i n}}=\underset{\Delta_{k, j}}{\operatorname{argmin}}\left(\left\|\epsilon_{k, j}+F_{k, j}^{L_{i n}}\right\|^{2}\right)$. 


\begin{tabular}{|c|c|c|c|c|c|}
\hline $\begin{array}{c}\text { Reference } \\
\text { Small Variation } \\
\text { Algorithm (a) }\end{array}$ & $\begin{array}{c}\text { 2bit.s } \mathrm{s}^{-1} / \mathrm{Hz} \\
\text { SNR } R_{r e f}=7.4 \mathrm{~dB} \\
8 \mathrm{PSK} \\
\text { OBO }=0.85 \mathrm{~dB}\end{array}$ & $\begin{array}{c}3 \text { bit. } \mathrm{s}^{-1} / \mathrm{Hz} \\
S N R_{\text {ref }}=11.85 \mathrm{~dB} \\
16 \mathrm{APSK} \\
\mathrm{OBO}=1.3 \mathrm{~dB}\end{array}$ & $\begin{array}{c}4 \text { bit.s } \mathrm{s}^{-1} / \mathrm{Hz} \\
S N R_{r e f}=16 \mathrm{~dB} \\
32 \mathrm{APSK} \\
\text { OBO=2dB }\end{array}$ & $\begin{array}{c}\text { 5bit.s } \text { - }^{-1} / \mathrm{Hz} \\
S N R_{\text {ref }}=20.3 \mathrm{~dB} \\
64 \mathrm{APSK} \\
\mathrm{OBO}=2.7 \mathrm{~dB}\end{array}$ & $\begin{array}{c}6 \text { bit. } \mathrm{s}^{-1} / \mathrm{Hz} \\
\text { SNR } R_{\text {ref }}=25.9 \mathrm{~dB} \\
128 \mathrm{APSK} \\
\mathrm{OBO}=2.9 \mathrm{~dB}\end{array}$ \\
\hline $\begin{array}{c}\text { Lower reduced } \\
\text { complexity SVA (b) }\end{array}$ & $S N R_{r e f}$ & $S N R_{r e f}$ & $S N R_{r e f}+0.15 \mathrm{~dB}$ & $S N R_{r e f}+0.15 \mathrm{~dB}$ & $S N R_{r e f}+0.4 \mathrm{~dB}$ \\
\hline $\begin{array}{l}\text { Intermediate reduced } \\
\text { complexity SVA (c) }\end{array}$ & $S N R_{r e f}+0.35 \mathrm{~dB}$ & $S N R_{r e f}+0.5 \mathrm{~dB}$ & $S N R_{\text {ref }}+0.65 d B$ & $S N R_{\text {ref }}+0.7 \mathrm{~dB}$ & $S N R_{r e f}+1 \mathrm{~dB}$ \\
\hline $\begin{array}{l}\text { Higher reduced } \\
\text { complexity SVA (d) }\end{array}$ & $S N R_{r e f}+0.35 \mathrm{~dB}$ & $S N R_{r e f}+0.5 \mathrm{~dB}$ & $S N R_{\text {ref }}+0.65 d B$ & $S N R_{\text {ref }}+1.3 \mathrm{~dB}$ & $S N R_{r e f}+1.3 \mathrm{~dB}$ \\
\hline $\begin{array}{l}\text { Memory Polynomial } \\
\text { pre-distorter (e) }\end{array}$ & $S N R_{\text {ref }}+0.4 \mathrm{~dB}$ & $S N R_{\text {ref }}+1.35 \mathrm{~dB}$ & $S N R_{\text {ref }}+3.3 d B$ & $S N R_{\text {ref }}+7.7 \mathrm{~dB}$ & $\begin{array}{c}\text { Not } \\
\text { Achievable }\end{array}$ \\
\hline $\begin{array}{c}\text { LUT } \\
\text { pre-distorter (f) }\end{array}$ & $S N R_{\text {ref }}+1.15 \mathrm{~dB}$ & $S N R_{r e f}+2.25 \mathrm{~dB}$ & $S N R_{\text {ref }}+1.8 d B$ & $S N R_{r e f}+1.9 \mathrm{~dB}$ & $S N R_{r e f}+5.3 \mathrm{~dB}$ \\
\hline
\end{tabular}

Fig. 3: Minimum SNR requested at receiver input for various target achievable spectral efficiency $\left(\right.$ bit.s $\left.s^{-1} / H_{z}\right)$

(a) Reference SVA $\left[M=L_{c}, L_{c}=101 T_{s}, R_{s}=\frac{1}{T_{s}}=38 \mathrm{MBaud}, N_{I T}=8\right]$, (b) reduced SVA $\left[M=3, L_{c}^{\prime}=\frac{L_{c}}{3}\right]$, (c) reduced SVA $\left[M=1, L_{c}^{\prime}=\frac{L_{c}}{3}\right]$ (d) reduced SVA $\left[M=1, L_{c}^{\prime}=\frac{L_{c}}{3}\right.$ and $F_{k, j}^{L_{i n}}$ approximated by LUTs with $L_{c}^{\prime \prime}=3$ for modulation schemes up to 32APSK and with a 3rd order Volterra model with $L_{c}^{\prime \prime}=1$ for 64APSK and 128APSK modulation schemes]

(e) Memory polynomial pre-distorter (fifth order Volterra coefficients, pre-distorter length $\left.L_{c}^{\prime}=11\right)$, (f) LUT pre-distorter $\left(L_{c}^{\prime}=3\right)$

The major drawback of this method is the complexity induced by the number of channel simulations involved by the iterative process; basically, three channel simulations are needed to compute the small variation $\Delta_{k, j}^{L_{i n}}$ to be applied to the symbol at step $j$ of iteration $k$; one simulation to evaluate the Euclidian distance $\epsilon_{k, j}$ and two additional simulations to assess the channel output variation function $F_{k, j}^{L_{i n}}$. Each channel simulation is applied to a sequence of $L_{c}$ symbols where $L_{c}$ denotes the channel model memory length. Therefore, if we define a Computation Unit (CU) as the amount of mathematical operations needed to output one symbol from the channel model depicted in Figure 4, the number of $\mathrm{CU}$ needed to pre-distort one symbol is equal to $N_{I T} \times L_{c} \times 3$ where $N_{I T}$ denotes the number of iterations.

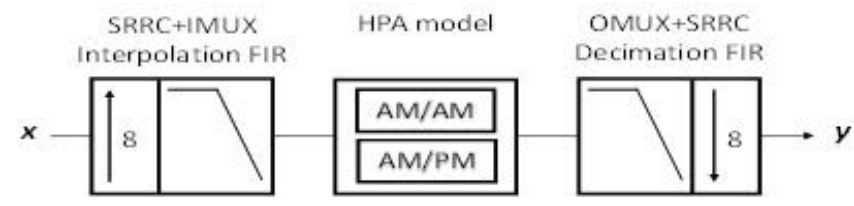

Fig. 4: SVA channel model

Preliminary approximations have been proposed in [4] to decrease the algorithm complexity by removing the channel simulations intended to evaluate $F_{k, j}^{L_{i n}}$ : one option is to tabulate it in look-up tables (LUTs), the other is to approximate the channel variation with a reduced Volterra model. In this paper, further optimizations have been brought for the purpose of prototyping SVA algorithm on a FPGA device:

1) Channel model optimization: The memory length $L_{c}^{\prime}$ of SVA embedded channel model depicted in Figure 4 may be decreased by truncating the IMUX, OMUX and shaping filter impulse response, keeping in mind that the achievable gain mainly relies on the accuracy of channel model used to assess the Euclidian distance $\epsilon_{k, j}$ and to a lesser extent on the accuracy of the channel output variation function $F_{k, j}^{L_{i n}}$ which can be approximated as proposed in [4]. Simulations have shown that a ratio $L_{c}^{\prime} / L_{c}=1 / 3$ has no significant impact on the residual $\mathrm{C} / \mathrm{I}$ measured on the received constellation.

2) Minimization of the channel simulation number per iteration: In the general formulation of SVA, only one symbol $x_{k, j}$ is updated at each step of iteration $k$. Thus, $L$ steps per iteration are requested to process a block of $L$ symbols. Considering that the channel model memory has a reduced finite length $L_{c}^{\prime}, L / M$ with $M=L_{c}^{\prime}$ pre-distorted symbols $x_{k, j}$ can be updated at each step $j$ of each $k$ iteration without impacting the performance since $x_{k, j}, x_{k, j+L_{c}^{\prime}}, x_{k, j+2 L_{c}^{\prime}}, \ldots$ can be assumed independant. Reducing $M$ down to 3 allows to drastically reduce the algorithm complexity without significant impact on the achievable gain. Still, reducing $M$ down to 1 results in a limited link budget degradation of $0.5 \mathrm{~dB}$ only. This latest option can be retained if the design rate performance shall take precedence over the algorithm accuracy.

3) Fixed-length framing: Processing symbols in fixedlength blocks whatever the modulation scheme not synchronized with PLFRAMES shall be favored for a hardware implementation. Extra symbols are appended at the beginning and at the end of each block $L$ of transmitted symbols to the extent of $K$ symbols, to mitigate side effects. Selection of $(L, K)$ framing parameters is a trade-off between framing efficiency, processing latency and side effects mitigation. $(L, K)=(4000,4096)$ values are considered for SVA hardware prototype.

The impact of these approximations on the benefit of SVA over state-of-art channel impairments mitigation techniques has been assessed using the EXIT charts based achievable rate $\left(\right.$ bit.s $\left.s^{-1} / \mathrm{Hz}\right)$ metric as function of the SNR available at receiver input. This study has been performed for all DVBS2X modulation schemes up to the 128APSK $140 / 180$ in a SNR range of $[-2, \ldots, 30] \mathrm{dB}$. Figure 3 shows which SNR is requested at the receiver input to achieve various spectral efficiencies, i.e. 2, 3, 4, 5, and 6 bit.s ${ }^{-1} / \mathrm{Hz}$, for all studied pre-distorters. These results clearly demonstrate that even when considering the most aggressive level of optimization 
(reduced SVA (e)), SVA outperforms the state-of-the art predistorters.

\section{B. Volterra based detectors}

An approach to mitigate channel distortions and enhance the link capacity is to implement advanced Volterra iterative equalizers in the receiver. This approach is referred to as turbo equalization and consists in enabling an iterative exchange of extrinsic soft information between the equalizer and the decoder. Indeed, according to [5], considering a baseband transmission, the non-linear channel can be modeled using a Volterra model with linear and non-linear terms. When Volterra model is truncated to a $3^{\text {rd }}$ order, the received time sample, $y_{k}$, can be written as

$$
y_{k}=\sum_{l} a_{k-l} K_{l}^{(1)}+\sum_{m, n, p} a_{k-m} a_{k-n} a_{k-p}^{*} K_{m, n, p}^{(3)},
$$

where $a_{k}$ are the transmitted complex symbols and $K_{l}^{(1)}$ and $K_{m, n, p}^{(3)}$ represent respectively the $1^{\text {st }}$ and $3^{\text {rd }}$ order kernels resulting from the Volterra modeling of the HPA and the different filters. Based on this model, both non-linear (MAP) and linear (MMSE) iterative detectors can be applied [7], enabling turbo-detection paired with Low-Density Parity Check (LDPC) decoding.

First, trellis-based iterative maximum a posteriori (MAP) symbol detection [12] is investigated. This turbo-equalization scheme offers high performance at the expense of higher complexity. The thorny point of the non-linear detector is the number of states in the trellis $M^{L_{c}^{\prime}}$ increasing with the modulation order $M$ and the number of $1^{\text {st }}$ order kernels taken into account in the truncated model $L_{c}^{\prime}$. A huge truncation on the channel length makes the receiver inefficient: the MAP Volterra detector will be only considered for low order modulation ( $Q$-PSK and 8-PSK) for feasibility reasons.

In order to employ higher modulation orders, linear MMSE detection was investigated with the aim of assessing the gain offered by MMSE turbo equalizers for different constellation sizes in a DVB-S2X channel. This study is based on the Volterra MMSE equalizers derived in [7]. Indeed, the estimated symbol, $\hat{a}_{n}$, at the MMSE equalizer output can be expressed as:

$$
\hat{a}_{k}=\sum_{i=-N_{2}}^{N_{1}} c_{i} y_{k-i}+b_{k}
$$

where $N_{1}$ and $N_{2}$ are respectively the post-cursor and precursor lengths. $\mathbf{c}_{\mathbf{i}}=\left[c_{-N_{2}}, \ldots c_{N_{1}}\right]$ and $b_{k}$ represent the solution that minimizes the MMSE criterion between the transmitted and estimated symbols $E\left[\left|\hat{a}_{k}-a_{k}\right|^{2}\right]$.

The MMSE solution considered in this study is an Exact MMSE taking into consideration the a priori information available in the equalizer input, resulting in a time variant solution. The computation of the solution as function of Volterra kernels is detailed on [7]. The performance and the complexity of MMSE Volterra equalizers depend on several parameters, namely the number of post-cursor and pre-cursor
MMSE coefficients $N_{1}$ and $N_{2}, L_{c}^{\prime}$ and $L_{3}$, the number of $1^{\text {st }}$ and $3^{\text {rd }}$ order Volterra kernels. These parameters have to ensure a good trade-off between complexity and equalization performance. The impact of the number of $3^{r d}$ order kernels, $L_{3}$, and the MMSE filter lengths is illustrated in Fig. 5.

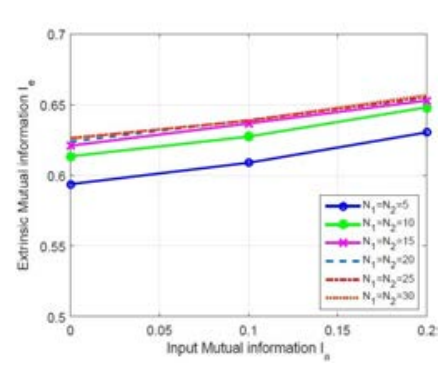

(a)

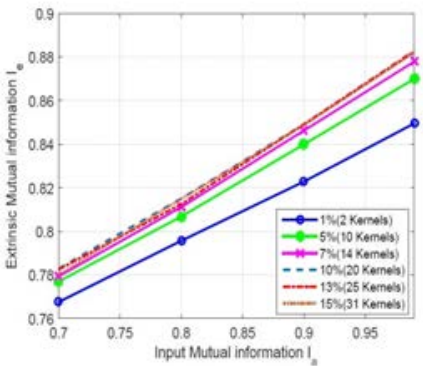

(b)
Fig. 5: Impact of equalization parameters on the EXIT chart performance: (a) MMSE filter lengths $\left(N_{1}, N_{2}\right)$ (b) Number of $3^{\text {rd }}$ order Volterra kernels, $L_{3}$.

As can be seen in Figure 5, the extrinsic information significantly increases with the MMSE filter lengths until saturation occurs at $N_{1}=N_{2}=20$, which corresponds to the performance limit of a Volterra MMSE equalizer. Similarly, the extrinsic information significantly increases with the number of $3^{\text {rd }}$ order Volterra kernels employed to perform the equalization, until reaching the performance limit at $L_{3}=20$. As a consequence, to ensure the best trade-off between complexity and equalization performance, we retain $N_{1}=N_{2}=20$ and $L_{3}=20$. Finally, the number of $1^{\text {st }}$ order kernels $L_{c}^{\prime}$ is computed to retain $99 \%$ of the signal energy. It corresponds to $L_{1}=15$ and $L_{2}=5$, where $L_{2}$ is the number of non causal kernels and $L_{1}$ the number of causal kernels.

\section{FTN signaling for spectral efficiency enhancement}

FTN signaling was first brought up by Mazo in [14]. It consists in an increase of the transmission rate resulting in spectral efficiency improvement at the expense of InterSymbol-Interference (ISI) introduction requiring advanced receivers. Then, the Nyquist criterion is no longer verified. With the $T$-orthogonal shaping filter $h_{e}(t)$ and $\alpha \in[0,1]$ the compression factor of the FTN transmission, the emitted signal is given by

$$
x(t)=\sum_{k} a_{k} h_{e}(t-k \alpha T)
$$

where $T$ is the filter period such as $h_{e}(k T)=0$, for $k \in$ $\mathbb{Z}^{*}$. Most recently, studies demonstrated the interest of such a signaling in linear context [15], including DVB-S2 context of comparison in [16].

Furthermore, FTN signaling has been investigated through non-linear channel. In [17], $M$-PSK FTN signaling is compared to an iso-capacity $N$-APSK Nyquist transmission, highlighting a peak-to-average power ratio (PAPR) reduction of the signal, reflecting an increased robustness against non-linear distortions. FTN signaling, also called time packing (TP), is 
paired with a MAP Volterra based detector in [3] and its relevance in the DVB-S2 context is conclusive. FTN signaling can take advantage of the model described in (3) and the associated Volterra based MAP detector considered in III-B

First, consider the transmission is achieved with a rate of 36Mbaud. Allowing FTN signaling highly increases the channel memory effect, leading to a higher truncated channel length required to take into account $85 \%$ of the introduced ISI energy. In Table I let's notice that $L_{c}^{\prime}=3$ is enough for Nyquist signaling whereas the considered FTN signaling $(\tau=0.7)$ requires a channel length $L_{c}^{\prime}=5$.

TABLE I: Truncated channel length $L_{c}^{\prime}$ required for modeling $85 \%$ of both linear and non-linear ISI introduced and associated binary achievable spectral efficiency $\gamma\left(\right.$ bit.s $\left.s^{-1} / \mathrm{Hz}\right)$ when Nyquist (NYQ) and faster-than-Nyquist (FTN) signaling $(\alpha=0.7)$.

\begin{tabular}{|c|c|c|c|}
\hline & 36Mbaud & 38Mbaud & 40Mbaud \\
\hline NYQ & $L_{c}^{\prime}=3, \gamma=0.90$ & $L_{c}^{\prime}=4, \gamma=0.95$ & $L_{c}^{\prime}=5, \gamma=1.0$ \\
\hline FTN & $L_{c}^{\prime}=5, \gamma=1.29$ & $L_{c}^{\prime}=5, \gamma=1.36$ & $L_{c}^{\prime}=5, \gamma=1.43$ \\
\hline
\end{tabular}

When rate enhancement is considered, the channel selectivity is increased for both Nyquist and FTN signaling. Table I shows off an associated increase of $L_{c}^{\prime}$ for Nyquist signaling whereas no change is observed for FTN signaling. The complexity increase due to time packing is no more detrimental when rate enhancement is considered and offers an improved spectral efficiency. This result motivates joint FTN sigaling with Volterra based detector and rate enhancement up to $40 \mathrm{Mbaud}$.

Last but not least, the Modulation and Coding (ModCod) diversity considering the additional degree of freedom $\alpha$ from FTN signaling increases the capacity granularity compared to a Nyquist signaling. When coding rate constraint from the standard DVB-S2X is taken into account, the optimal capacity improvement thanks to FTN is more relevant, as depicted in Figure 6.

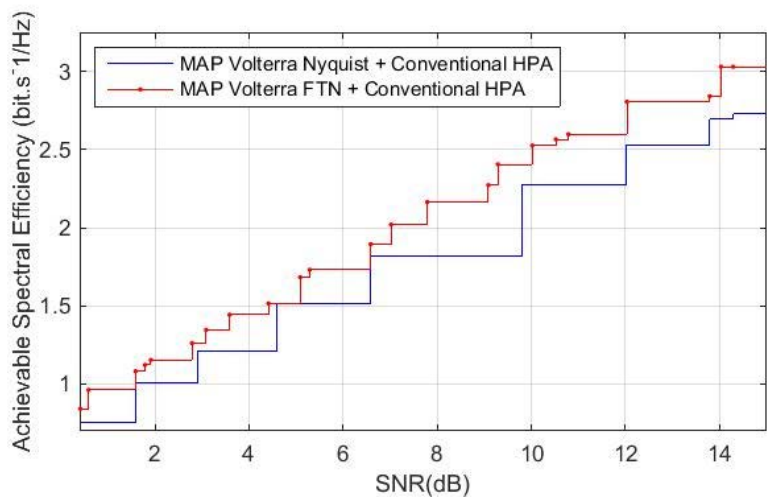

Fig. 6: Volterra based MAP detector capacity considering the coding rate constraint for Nyquist and FTN (dotted) signaling.

The mean capacity improvement in the SNR range from $0 \mathrm{~dB}$ to $15 \mathrm{~dB}$ is $11 \%$ and is up to $20 \%$ on some targeted SNR with no complexity increase thanks to FTN signaling.

\section{SC-OFDM waveform and frequency equalization}

As an alternative of DVB-S2X air interface, SC-OFDM is a multi-carrier transmission scheme OFDM with Discrete Fourier Transform (DFT) precoding [18]. This precoding allows SC-OFDM to be a single carrier transmission scheme with the same order of signal dynamics, e.g. PAPR, as Time Division Multiplexing (TDM) signal used in DVB-S2X. Moreover, introduction of root raised cosine shaping in frequency domain between DFT precoding and Inverse DFT (IDFT) lowers the side-lobes of the resulting time domain generated waveform enabling similar PAPR characteristic of TDM signal [19]. This particular spectral shaping, described on Figure 7, characterizes the EW-SC-OFDM signal. Thereby, SC-OFDM and EW-SC-OFDM are well fitted for satellite transmission considering their reduced PAPR. These waveforms also benefit from low complexity frequency domain equalization offered by OFDM signaling. In this study, sizes of DFT and IDFT are respectively 1024 and 8192 resulting in the oversampling $N_{s}=8$. Introduction of cyclic prefix, related to channel memory length $\left(L_{c}\right)$, reduces the spectral efficiency of about $3 \%$. However, note that this study shows a spectral efficiency reduction from about $7 \%$ when the cyclic prefix is not inserted. Finally, roll-off factor of SRRC window for EW-SC-OFDM meets the specified 0.1. At a glance, SCOFDM (EW-SCOFDM) signaling is simply a new option for the shaping filter of DVB-S2X air interface at the transmitter. Thus, signal before DFT carry a sequence of DVB-S2X physical layer frames. At the receiver, this technique provides an equivalent to the matched filter all in carrying a MMSE spectral equalization. Here, MMSE filter is based on analytical spectral response of on board channelizing. Note that future pilot insertion for channel estimation will lead to a loss below $5 \%$ of the spectral efficiency.

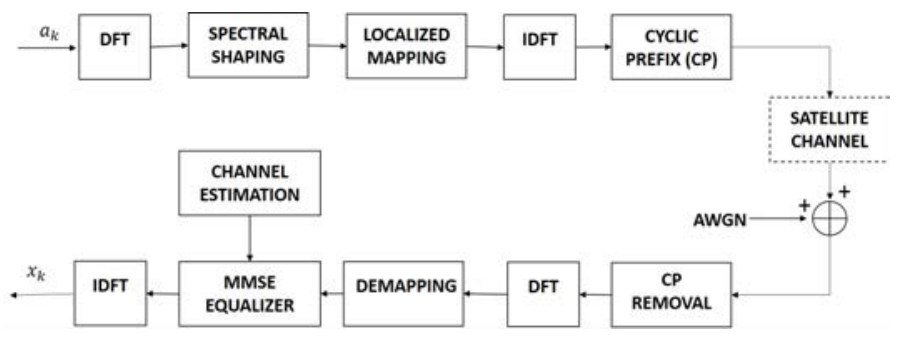

Fig. 7: EW-SCOFDM scheme

Optima of spectral efficiency have been obtained increasing the rate, testing range of IBO over the spectrum of SNR and modulations for SC-OFDM and EW-SC-ODM. The higher spectral efficiency has been reached considering a linearized amplifier and a symbol rate of $40 \mathrm{MBauds}$. As expected w.r.t the linear equalization, the use of nonlinearized amplifier degrades the spectral efficiency compared to the linearized amplifier from about $50 \%$. Then, from 36 M Bauds to $40 \mathrm{MBauds}$, spectral efficiency shows a gradual increase especially considering high SNR. Above 40MBauds, spectral efficiency decreases dramatically. Regarding IBO, the higher the SNR is, the higher the back-off has to be. Finally, below 
$40 \mathrm{MBauds}$, the EW-SC-OFDM spectral efficiency is higher than the SC-OFDM one. However, this difference decreases with the rate augmentation. At $40 \mathrm{MBauds}$, SC-OFDM spectral efficiency meets EW-SC-OFDM one, except for some SNR range where EW-SC-OFDM is still higher especially with unexpected 0.05 roll-off. Compared to the standard LMS equalization, at $36 \mathrm{MBauds}$, EW-SC-OFDM shows identical spectral efficiency minus the cyclic prefix tax. As rate increase, EW-SC-OFDM and SC-OFDM exceed standard LMS spectral efficiency and give an estimation of the best spectral efficiency obtained with a linear equalization using a linear amplifier.

\section{REsults}

\section{A. DVB-S2X reference}

The transmission meets the specification detailed in II. In order to benefit the capacity improvement promised by DVB-S2X, the considered HPA is linearized (AM/AM curve in Figure 1-a). At the receiver, after matched filtering and down-sampling, a 16-taps LMS equalizer described in [2] is implemented, as suggested in [1]. This receiver counteracts the linear distortions introduced in the payload but does not take into account the non-linear distortions. In Figure 9 and 10, the DVB-S2X promises considering a linear channel, called "AWGN channel" gives the full potential of the standard. When the LMS equalizer is implemented to overcome the distortions introduced by the linearized amplifier, part of the potential is regained. Capacity improvement is still possible, as highlighted by the gap between "AWGN channel" and "DVB-S2X reference" curves. At the expense of a linearized amplifier and an improved receiver by LMS implementation, the full potential of the standard is not achieved. Do advanced techniques improve the satellite capacity when non-linearized amplifier is considered?

\section{B. Comparison results}

In this paragraph, we first oppose the performance of the different detailed techniques in Section III. The optimal achievable spectral efficiency was computed for each transmission scheme thanks to the EXIT charts computation described in Section II-C. The optimized rates for each advanced techniques are $R_{s}=40 \mathrm{MBauds}$ except for SVA where $R_{s}=38 \mathrm{MBauds}$. Figure 8 depicts the performance of the proposed advanced techniques:

- SVA pre-distorter offers an increased spectral efficiency compared to the DVB-S2X reference in the range of low SNR. When higher SNR is considered (from $10 \mathrm{~dB}$ to 30dB), SVA pre-distorter achieves the best performance and significantly improves the DVB-S2X reference capacity.

- In the range of low SNR (from 0dB to 10dB), FTN signaling paired with a Volterra based MAP detector offers the highest performance. Its improved capacity thanks to compression factor on low order constellation is relevant compared to the DVB-S2X reference. When high SNR range is considered, FTN Volterra based MAP detection is indeed no more relevant since the modulation order for feasibility is limited to 8PSK.

- Nyquist signaling with Volterra based MAP detection is not relevant since its performance is lower than the DVBS2X reference. This advanced technique is not kept for the following of this paper.

- Nyquist signaling with Volterra based MMSE detection is relevant when higher SNR are taken into account making FTN+Volterra based MAP detection not feasible anymore. However its performance with regard to the DVB-S2X reference is not relevant enough and is not considered in the following of this paper.

- SC-OFDM signaling with frequency domain equalization is relevant when high order modulations are used in the range of high SNR. Its limited performance is explained since we only took into account the linear distortion for detection. A Volterra model in frequency domain would be more relevant and would allow transmission with a conventional amplifier.

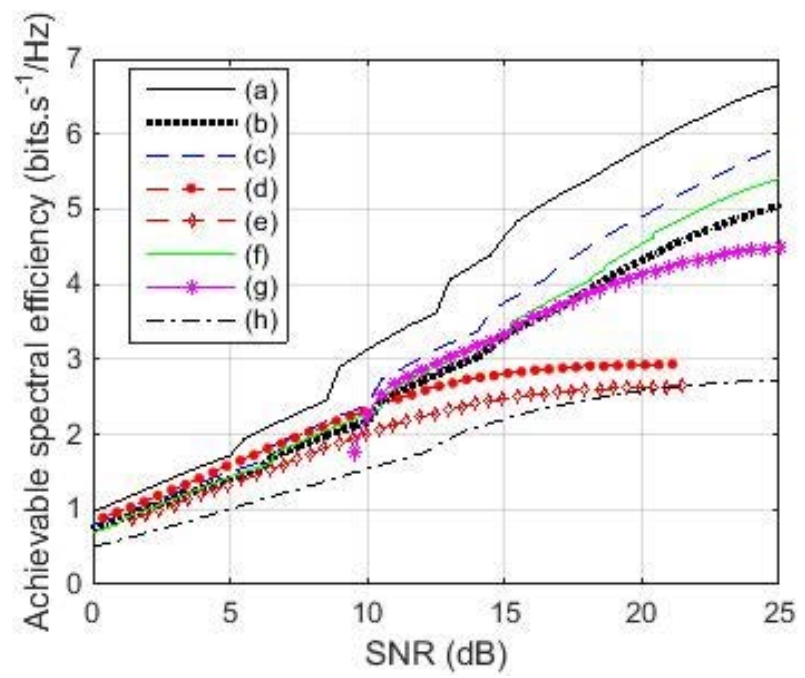

Fig. 8: Achievable Spectral Efficiency $\left(\right.$ bit.s $\mathrm{s}^{-1} / \mathrm{Hz}$ ) Vs SNR (dB)

(a) DVBS2X in AWGN channel (b) DVBS2X Reference : On board HPA linearization and 16-tap receiver equalization (c) SVA pre-distortion with conventional on-board HPA (d) MAP Volterra FTN signaling with conventional on-board HPA (e) MAP Volterra receiver with conventional on-board HPA (f) SC-OFDM Signaling (g) Turbo MMSE equalization with conventional on-board HPA (h) DVBS2X in dispersive channel (on-board conventional HPA) and no distortion mitigation

Then, for conclusion, we select the two most relevant techniques for low SNR and the most relevant for high SNR. In Figure 9 and Figure 10, we take into account the standard coding rates of which FTN signaling takes advantage with its inherent increased granularity.

- Figure 9: FTN paired with Volterra based MAP detector offers an average increase of the DVB-S2X reference spectral efficiency of $19 \%$ on the range of SNR [0.5dB to $10 \mathrm{~dB}$ ]. In the same time, the SVA increased spectral 
efficiency considering the DVB-S2X reference in this range of SNR is $7 \%$.

- Figure 10: SVA pre-distorter improves the average capacity by $11 \%$ with regard to the DVB-S2X reference in the range of SNR [10dB to $30 \mathrm{~dB}$ ].

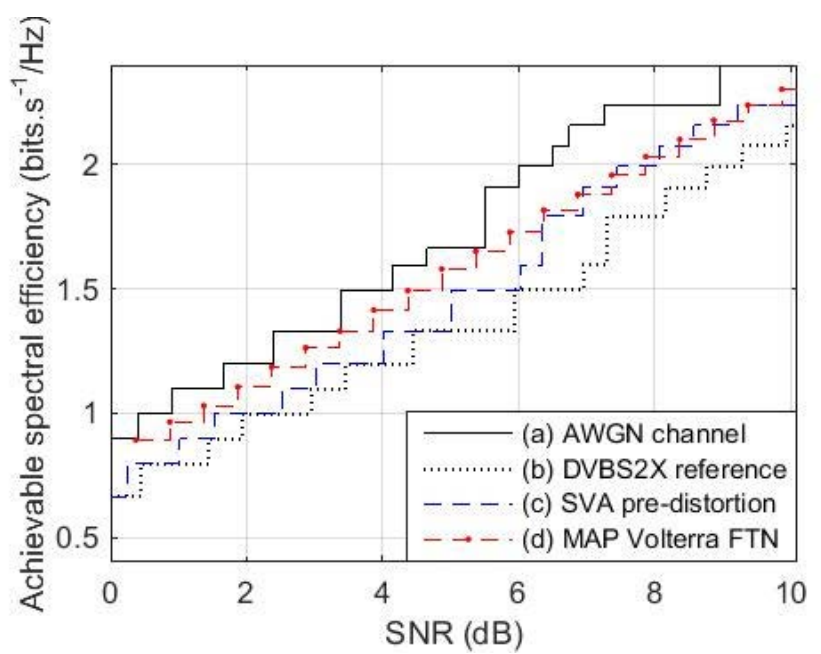

Fig. 9: Achievable Spectral Efficiency (bit.s $\left.{ }^{-1} / H z\right)$ Vs SNR (dB) - Range of low SNR

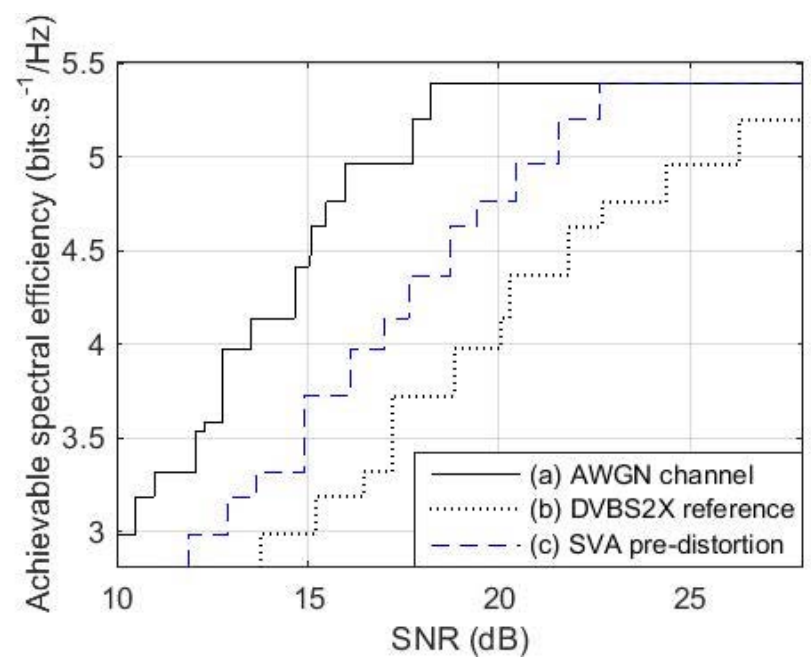

Fig. 10: Achievable Spectral Efficiency $\left(\right.$ bit.s $\left.{ }^{-1} / H z\right)$ Vs SNR (dB) - Range of high SNR

\section{CONCLUSION}

In this paper, advanced techniques at the transmitter and/or the receiver demonstrate significant capacity improvement compared to the DVB-S2X reference. Moreover, it allows transmission with a conventional HPA of which the energy efficiency is better than a more expensive linearized HPA. On one hand, in the range of low SNR, this paper shows off an improved capacity of $19 \%$ thanks to FTN paired with Volterra based MAP detection. On the other hand, in the range of high SNR, SVA pre-distorter allows an increase of the capacity of $11 \%$. In further studies, transmissions in wideband transponders, of which the memory effects are higher, will be considered. Volterra based frequency model will be considered for SC-OFDM equalization.

\section{REFERENCES}

[1] ETSI, Digital Video Broadcasting (DVB): Second generation framing structure, channel coding and modulation systems from Broadcasting, Interactive Services, News Gathering and other broadband satellite applications. Part 2: DVB-S2 Extensions (DVB-S2X), ETSI EN 302 307-2 V1.1.1, 10/2014.

[2] J.G. Proakis, M. Salehi, Digital Communications, 5th Edition, McGrawHill, 2008 - Chapter 10.

[3] A. Piemontese, A. Modenini, G. Colavolpe and N.S. Alagha, "Improving the Spectral Efficiency of Nonlinear Satellite Systems through TimeFrequency Packing and Advanced Receiver Processing”, IEEE Transactions on Communications Theory, vol. 61, No. 8, Aug. 2013

[4] T. Deleu, M. Dervin, K. Kasai, and F. Horlin, Iterative Predistortion of the Nonlinear Satellite Channel, IEEE Transactions on Communications, vol. 62, no. 8, Aug. 2014

[5] S. Benedetto, E. Biglieri, and R. Daffara, Modeling and performance evaluation of nonlinear satellite links-a volterra series approach, IEEE Transactions on Aerospace and Electronic Systems, vol. AES-15, no. 4, pp. 494 507, july 1979

[6] . G. Colavolpe, A. Piemontese, "Novel SISO Detection Algorithms for Nonlinear Satellite Channels", IEEE Wireless Communications Letters, Feb. 2012.

[7] B. Benammar, N. Thomas, M.L. Boucheret, C. Poulliat, M. Dervin "A comparison of iterative receivers for the non linear satellite channel"IEEE 16th International Workshop on Signal Processing Advances in Wireless Communications (SPAWC), 2015

[8] Stephan Ten Brink, "Convergence Behavior of Iteratively Decoded Parallel Concatenated Codes", IEEE Transactions On Communications, vol. 49, No. 10, October 2001

[9] Alexei Ashikhmin, Gerhard Kramer and Stephan ten Brink, "Extrinsic Information Transfer Functions: Model and Erasure Channel Properties" IEEE Transactions On Information Theory, vol. 50, No. 11, November 2004

[10] D. Morgan, Z.Ma, J. Kim, M. Zierdt, and J. Pastalan, Ageneralized memory polynomial model for digital predistortion of $R F$ power amplifiers, IEEE Trans. Signal Process., vol. 54, no. 10, pp. 3852-3860, Oct. 2006.

[11] E. Casini, R. D. Gaudenzi, and A. Ginesi, DVB-S2 modem algorithms de sign and performance over typical satellite channels, International Satellite Communictions Networking, vol. 22, no. 3, pp. 281-318, May/Jun. 2004.

[12] L. R. Bahl, J. Cocke, F. Jelinek, and J. Raviv , ”Optimal Decoding of Linear Codes for Minimizing Symbol Error Rate”, IEEE Transactions on Information Theory, March 1974

[13] G. David Forney, ”Maximum-Likelihood Sequence Estimation of Digital Sequences in the Presence of Intersymbol Interference ", IEEE Transactions on Information Theory, vol. IT-18, No. 3, May 1972

[14] J.E. Mazo, "Faster than Nyquist Signaling”, Bell Syst. Tech. J., vol. 54, pp, 1451-1462, Oct. 1975

[15] F. Rusek and J. B. Anderson, "Constrained capacities for faster than Nyquist signaling”, IEEE Trans. Inf. Theory, vol. 55, no. 2, pp. 764-775 Feb. 2009.

[16] N. Pham, J. B. Anderson, F. Rusek and J. M. Freixe , "Exploring Fasterthan-Nyquist for Satellite Direct Broadcasting”, 31st AIAA International Communications Satellite Systems Conference, 2013

[17] J.A. Lucciardi, N. Thomas, M.L. Boucheret, C. Poulliat, G. Mesnager, "Trade-Off Between Spectral Efficiency Increase and PAPR Reduction When Using FTN Signaling: Impact Of Non Linearities", IEEE International Conference on Communications (ICC), 2016

[18] H.G. Myung, J. Lim, D. J. Goodman, ”Single carrier FDMA for uplink wireless transmission”, IEEE Vehicular Technology Magazine, vol. 1, iss. 3, 2006

[19] O. Mauritz and B. Popovic, "Optimum Family of Spectrum-Shaping Functions for PAPR Reduction of DFT-Spread OFDM Signals", IEEE 64th Vehicular Technology Conference, 2006 